\title{
Encoding-based brain-computer interface controlled by non-motor area of rat brain
}

\author{
LANG YiRan*, DU Ping \& SHIN Hyung-Cheul \\ Department of Physiology, College of Medicine, Hallym University, Chuncheon 200-702, Republic of Korea
}

Received March 20, 2010 ; accepted May 25, 2011

\begin{abstract}
As the needs of disabled patients are increasingly recognized in society, researchers have begun to use single neuron activity to construct brain-computer interfaces (BCI), designed to facilitate the daily lives of individuals with physical disabilities. BCI systems typically allow users to control computer programs or external devices via signals produced in the motor or pre-motor areas of the brain, rather than producing actual motor movements. However, impairments in these brain areas can hinder the application of BCI. The current paper demonstrates the feasibility of a one-dimensional (1D) machine controlled by rat prefrontal cortex (PFC) neurons using an encoding method. In this novel system, rats are able to quench thirst by varying neuronal firing rate in the PFC to manipulate a water dish that can rotate in 1D. The results revealed that control commands generated by an appropriate firing frequency in rat PFC exhibited performance improvements with practice, indicated by increasing water-drinking duration and frequency. These results demonstrated that it is possible for rats to understand an encoding-based BCI system and control a 1D machine using PFC activity to obtain reward.
\end{abstract}

brain-computer interface, prefrontal cortex, rat, one-dimensional, single neuron recording

Citation: $\quad$ Lang Y R, Du P, Shin H C. Encoding-based brain-computer interface controlled by non-motor area of rat brain. Sci China Life Sci, 2011, 54: 841-853, doi: 10.1007/s11427-011-4214-6

Brain-computer interfaces (BCI) enable patients with motor-related disabilities [1] and healthy individuals to manipulate prostheses and computer programs directly via their brain activity, with invasive [2,3] or non-invasive systems [4-6]. Recently, several groups have investigated the possibility of cortically-controlled neural prostheses [7-15]. Neuronal activity in the premotor, primary motor and posterior parietal cortical areas of non-human primates has been successfully used to control motor tasks [16] in BCI systems. Studies have shown that primates can learn to reach and grasp virtual objects by controlling a robot arm through a closed-loop brain-machine interface using mathematical modeling to extract several motor parameters from the electrical activity of frontoparietal neuronal ensembles [17]. In such a system, monkey primary motor neurons are decoded

*Corresponding author (email: langyiran@sina.com) into a signal that can move a computer cursor to a new position in two-dimensional space [18]. These methods transform neuronal population signals in the motor areas into real-time movements of prosthetic devices. Unfortunately, this type of motor information cannot be obtained in patients with disorders affecting motor-related brain areas. It is well established that the prefrontal cortex (PFC) is associated with behavioral flexibility, working memory, planning, spatial navigation, and goal-directed behavior [19, 20]. Despite the important functions of the PFC, it has never been utilized in BCI systems.

Increased understanding of the way in which the brain represents movement would facilitate the design of appropriate decoding algorithms, which form the basis of current BCI research. Nonetheless, we hypothesize that it is not necessary to fully decode neural signals. Simple models and 
encoding algorithms may be sufficient for the neural control of devices, since the brain is a complex learning system that can learn new arbitrary tasks with appropriate training. In the current study, we developed a relatively simple algorithm for rats to control a BCI system using neuronal activity in the PFC.

\section{Materials and methods}

\subsection{Implantation surgery and electrophysiology}

Experiments were approved by the Hallym University Animal Care and Use Committee. Sprague-Dawley rats (230-280 g) were used in this study, obtained from the Experimental Animal Center of Orient Bio Co., Seongnam-si, Korea. The environment of the breeding room was maintained at $(23 \pm 2)^{\circ} \mathrm{C}$ and relative humidity was $50 \% \pm 10 \%$. Artificial lighting was used to light the room for $12 \mathrm{~h}$ per day. For surgery, rats were anesthetized with ketamine (i.m., Yuhan, Seoul, Korea; $100 \mathrm{mg} \mathrm{kg}{ }^{-1}$ ) and xylazine (Bayer Korea, Seoul, Korea; $5 \mathrm{mg} \mathrm{kg}{ }^{-1}$ ). A relatively large craniotomy (2-3 $\mathrm{mm}$ in diameter) was made bilaterally over the PFC. The PFC region $(2.7 \mathrm{~mm}$ anterior to bregma, 0.5$1.0 \mathrm{~mm}$ lateral from midline, $1.8-2.0 \mathrm{~mm}$ ventral to the dorsal surface of the brain surface) of the left and right PFC was identified according to a previously published rat brain map [21], and one or two multi-wire recording electrodes (tungsten microwire, A-M systems, USA; $40 \mu \mathrm{m}$ in diameter, teflon-coated) were lowered into the cortex, targeting layer IV of the PFC (Figure 5E) with a hydraulic device (Narishige, Tokyo, Japan). The custom-made 8-channel electrode array $(2 \times 4)$ consisted of two bundles of four microwires. Each bundle was separated by approximately $1 \mathrm{~mm}$ and the interval between adjacent microwires was approximately $50 \mu \mathrm{m}$. After verification of single neuron activity, the base of the electrode bundles was covered with gel-foam and cemented to the pre-screwed anchors with dental resin. The ground wire was attached to one of the screws penetrating the skull, $3 \mathrm{~mm}$ away from electrode bundles.

After surgery, a recovery period at least one week was allowed before experimentation. Forty-eight hours prior to the experiment, rats were deprived of water to increase thirst. Each rat was placed into the experimental cage and the rat was implanted with the electrode connector to check for the presence of neurons using a data acquisition system (MAP, Plexon Inc., Dallas TX, USA) for online multi-channel spike sorting and unit recording. The MAP system recorded up to 64 continuous analog signals at $40 \mathrm{kHz}$ using National Instruments Data Acquisition (NI DAQ, National Instruments Co., Texas, USA) devices. A pre-amplifier delivered improved signal-to-noise (10 times of gain) and jumperable analog low-cut filter selection to a computer. For spike recording, low-cut of bandpass was $150 \mathrm{~Hz}$ and high-cut was $3 \mathrm{kHz}$. The signals were improved digitally by applying 20000 times the gain with the computer system.

The raw signals were overlapped every $800 \mu$ s with a window discriminator (Figure 1A), and sorted on the basis of amplitude and peak-to-peak distance. Power spectral density analysis was used to rule out the environmental noise (for example, $30 \mathrm{~Hz}$ power consumption). Signals with a signal-to-noise ratio lower than 3:1 were discarded. Autocorrelograms were examined off-line after sorting single cell signals. If no clear refractory period (1-2 ms) was detected in the autocorrelogram, additional feature combinations were examined to subdivide the cluster further until a clear refractory period was presented in the autocorrelogram [22]. Templates of the units were prepared and saved separately for each rat for later use.

\subsection{Algorithm}

Among simultaneously recorded units, two (N1 and N2) were selected and their activities were converted in every $200 \mathrm{~ms}$ to commands through a triple threshold comparator algorithm (Figure 1B). Pre-processing was applied to $40 \mathrm{~s}$ of data to calculate the mean firing rate and standard deviation (SD) under normal resting conditions. The activity of N1 and N2 neurons was measured to serve as baseline and converted to four activity ranges around the mean firing rates $(\mathrm{M} \pm(0.5 \times \mathrm{SD}))$. The four firing ranges $(\geqslant(\mathrm{M}+0.5 \times$ $\mathrm{SD})+1, \mathrm{M} \sim \mathrm{M}+0.5 \times \mathrm{SD}, \mathrm{M}-0.5 \times \mathrm{SD} \sim \mathrm{M}-1, \leqslant(\mathrm{M}-0.5 \times \mathrm{SD})-1)$ of $\mathrm{N} 1$ and $\mathrm{N} 2$ were normalized by assigning four command values for clockwise (CW; 3, 2, 1, 0) and counter-clockwise $(\mathrm{CCW} ; 0,-1,-2,-3)$ rotation of water dish. N1 was responsible for $\mathrm{CW}$ and N2 was responsible for CCW movement. Machine control commands were generated by the values resulting from the addition of $\mathrm{CW}$ and $\mathrm{CCW}$ commands. The i80196 microprocessor thus received seven types of commands $(3,2$, and 1 for $\mathrm{CW} ;-1,-2$, and -3 steps of rotation for $\mathrm{CCW}$; and 0 for STOP) from the control PC, and executed them accordingly. One step of rotation in any direction turned the wheel exactly $14.5^{\circ}$, two steps turned it $21.5^{\circ}$, and three steps turned it $28.5^{\circ}$, respectively. When no turn (STOP) command was received, the stepper motor's magnetic force strongly grabbed the axis of rotation, so it was impossible to rotate the wheel by external force from animals. A 'FLUSHING' command generates a compelled rotation of the wheel when the STOP command was continuously transmitted for $5 \mathrm{~s}$. This was implemented to disable any long periods of water drinking, which could result in the early termination of the session. The FLUSHING command rotated the wheel in the opposite direction of the previous rotation, by the number of degrees corresponding to three steps.

\subsection{Experimental procedures}

In the animal chamber of the BCI system, a rat was placed 
in front of a horizontally rotating cylinder $(10.0 \mathrm{~cm}$ in diameter) with circular dish (7 $\mathrm{cm}$ in diameter) containing water in the black painted quadrant on it (Figure 1A). The height of the circular dish was the same as that of the floor of the chamber. Drinking was made possible by maintaining the water dish in front of the rat. Three narrow flat steel platforms ( $4 \mathrm{~cm}$ long, $5 \mathrm{~mm}$ wide) were placed at the floor edge to prevent the rats grasping the dish with their forepaws. An experimental session would be initiated only if a rat showed continuous (more than $40 \mathrm{~s}$ without distraction) water-seeking behavior three times within 3 min.

A session of machine control trials (T) for 6 min was followed by a 6 min rest session (REST) in which water dish was blocked by a white paper wall. This sequence of wheel control and rest period was repeated 3-6 times until the rat showed no interest in drinking. After the last trial, rats were allowed access to food pellets (free food period, FF; Figure 3C and D, Figure 4D and E) but water access was blocked. Water was then offered freely for 6 min (free water period, FW; Figure 3C and D, Figure 4D and E) respectively. The actual wheel rotations were switched off but unit recordings and command generation were recorded during FF and FW. A video camera positioned $40 \mathrm{~cm}$ above the mechanical apparatus recorded all experimental sessions to monitor the rats' behavior. AVI format files were recorded at $30 \mathrm{~Hz}$ and compressed using Microsoft MPEG-4 video codec V2 (compression rate of 100\%). Synchronization between the data acquisition system and video recordings was achieved using a light emitting diode that lit up at the start of the neuronal spike recording. The timings of the beginning and the end of water drinking were manually marked by frame-by-frame reviews of the video recordings by the investigators, with an accuracy of \pm 1 video frame. These timings were input into the computer to correlate neuronal activity during the water drinking (WD) and nonWD periods.

\subsection{Data analysis}

Statistical analysis was carried out using Graphpad Prism 4.0 (GraphPad Software, Inc., USA) and InStat 3.05 (GraphPad Software, Inc., USA). One way repeatedmeasures of analysis of variance (ANOVA) was used to test the statistical significance of differences within groups of individual units between different behavioral conditions. All pair-wise multiple comparison procedures (Student-Newman-Keuls method) were also applied to isolate the group or groups that differed significantly from the others. In every case, $P$-values less than 0.05 were considered to indicate statistical significance.

Firing frequency of neuronal signals was binned every 10 $\mathrm{ms}$ (number of spikes in every $10 \mathrm{~ms}$ was converted into count $\mathrm{s}^{-1}$ ) and no smoothing or overlapping was applied after calculation.

\subsection{Histological identification}

For the histological analysis, animals were anesthetized with sodium pentobarbital (30 mg kg-1, i.p.) and perfused transcardially with $0.1 \mathrm{~mol} \mathrm{~L}^{-1}$ phosphate-buffered saline (PBS, pH 7.4) followed by $4 \%$ paraformaldehyde in $0.1 \mathrm{~mol}$ $\mathrm{L}^{-1}$ phosphate-buffer ( $\mathrm{PB}, \mathrm{pH}$ 7.4). Brains were removed and postfixed in the same fixative for $6 \mathrm{~h}$. The brain tissues were cryoprotected by infiltration with $30 \%$ sucrose overnight. Thereafter, frozen tissues were serially sectioned with a cryostat (Leica, Germany) into $30-\mu \mathrm{m}$ coronal sections, then collected into six-well plates containing PBS. Cresyl violet staining was conducted to obtain a clear view of target area. The sections were mounted on gelatin coated microscopic slides. Cresyl violet (CV) acetate (Sigma, MO) was dissolved at $1.0 \%(\mathrm{w} / \mathrm{v})$ in distilled water, and glacial acetic acid was added to $t$ he solution. Before and after staining for $2 \mathrm{~min}$ at room temperature, the sections were washed twice in distilled water, dehydrated by placing successively for $2 \mathrm{~h}$ in $50 \%, 70 \%, 80 \%, 90 \%, 95 \%, 100 \%$ ethanol baths at room temperature, and finally mounted with Canada balsam (Kanto, Tokyo, Japan; Figure 5E) [23].

\section{Results}

In total, we analyzed experimental results from 56 trials, 56 rest sessions, as well as FW, FF, and FAR sessions, in 16 rats. The attention of rats was measured by focusing time when the dish was located in front of animals at the minimum distance for the dish to be out of their reach; this distance differed according to the reaching ability of animals. The duration of recording summed to a total of more than $600 \mathrm{~min}$. Rats were allowed $30 \mathrm{~min}$ to adapt to the environment before the experiment began.

Figures 1 and 2 show an example of machine control. Fourteen single units were simultaneously recorded, and units $7 \mathrm{~b}$ and $7 \mathrm{~d}$, isolated using a microwire, were used for machine control (Figure 1A and B). As in Chapin's (1999) paradigm [24], we used percentage of success in obtaining water reward as a measure of efficiency. Overall wheel rotations in a trial comprise repetitions of two periods: the WD and no-WD (NWD) periods. Video analyses revealed clear increases of WD frequency and total WD duration as trials were repeated from T1 to T5 (Figure 1C). The total WD duration in the first time-period (T1) was less than $1 \%$ of 6 min, while that at T6 was more than $26 \%$. This performance elevation was mainly due to increases in WD frequency $((r)=0.9950)$, since averaged WD durations (in s, T1: 1, T2: $1.57 \pm 0.26$, T3: $2.24 \pm 0.38$, T4: $2.70 \pm 0.30$, T5: $3.12 \pm 0.38)$ were not significantly different among five trials $(P=0.0673$, Kruskal-Wallis Test). WD events occurred intermittently at T2 and T3, but were spread over the entire 6 min period of T5 (Figure 1D).

Wheel rotations were controlled by $\mathrm{CW}, \mathrm{CCW}$, and 

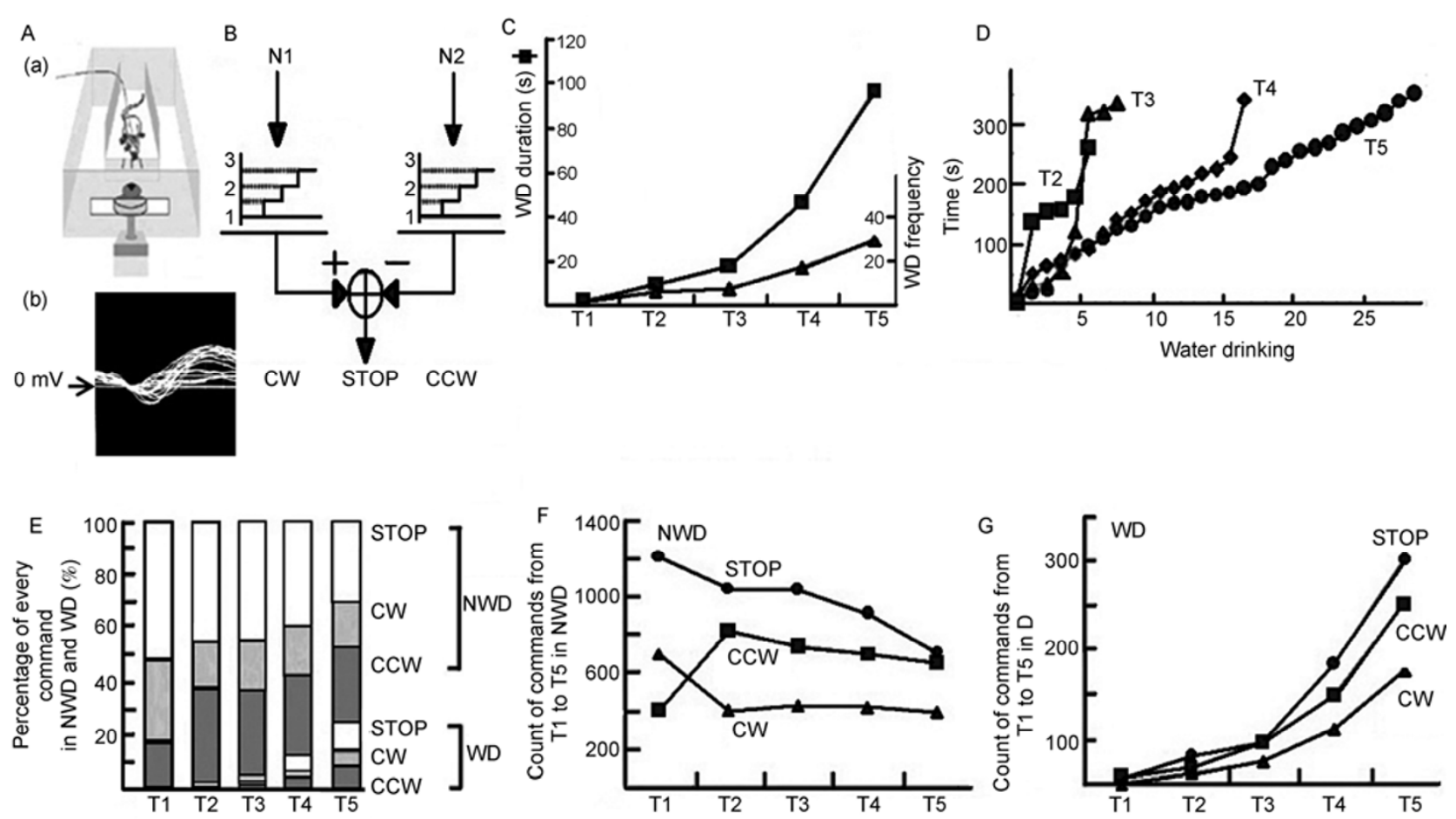

Figure 1 A, (a) Setup of 1-D system. A computer interface in which rats could freely move in a chamber, facing a water containing dish in the same plane of chamber. (b) Sample signals overlapped in a window discriminator. B, Triple-threshold algorithm for generating rotating command after comparing activities of two neurons. N1, neuron 1; N2, neuron 2. CW, clockwise; CCW, counterclockwise. C, WD duration and WD frequency changed across trials. D, Accumulative graph showing WD in every trial. E, Proportional movement-command distribution during NWD and WD. F, Count of movement-command changes over trials during NWD. G, Count of movement-command changes over trials during WD.

STOP commands. The STOP command constituted 52.17\% of all commands generated during $\mathrm{T} 1$, but this ratio was reduced to $41.24 \%$ in T5 (Figure 1E). Although STOP commands during NWD period from T1 to T5 were gradually decreased (Figure 1F), those during the WD period were gradually increased as trials progressed (Figure 1G). STOP commands in the NWD period varied inversely to the frequency of WD from T1 to T5 $((r)=-0.9804)$. During NWD periods from $\mathrm{T} 1$ to $\mathrm{T} 5$, changes in the frequencies of $\mathrm{CW}$ and CCW commands were observed in opposite directions $((r)=-0.9147)$. CW rotations were common at $\mathrm{T} 1$, but $\mathrm{CCW}$ rotations were dominant over $\mathrm{CW}$ rotations from $\mathrm{T} 2$ to T5 (Figure 1F). During WD periods, the frequency of $\mathrm{CW}, \mathrm{CCW}$, and STOP commands all increased (Figure 1G), and changes in the frequencies of these commands were strongly related $((r)>0.99)$ to changes of both WD frequency and total WD duration.

Neural activity of both $7 \mathrm{~b}$ and $7 \mathrm{~d}$ prior to command generation were reflected the operation of the triple-step threshold comparator algorithm (Figure 1B). $7 \mathrm{~b}$ and $7 \mathrm{~d}$ were set to encode $\mathrm{CW}$ and $\mathrm{CCW}$ commands, respectively. Triple-step thresholds for unit $7 \mathrm{~b}$ were set to $29,27,25$ in Hz (CW threshold values: above 29: 3, 27-28: 2, 25-26: 1, below 25: 0) and those of $7 \mathrm{~d}$ were to $27,23,19$, in $\mathrm{Hz}$ (CCW threshold values: above 27: 3, 23-26: 2, 19-22: 1, below 19: 0). In the $200 \mathrm{~ms}$ period prior to either $\mathrm{CW}$ or $\mathrm{CCW}$ command generation, both neurons behaved antago- nistically (Figure 2A and B). For the T5 WD period, neuron $7 \mathrm{~b}$ exhibited elevated activity $((35.95 \pm 1.72) \mathrm{Hz})$, generating three steps of $\mathrm{CW}$ rotation (i.e., threshold value 3 for $\mathrm{CW}$ ), whereas neuron $7 \mathrm{~d}$ exhibited suppressed activity $((18.69 \pm 1.05) \mathrm{Hz}$, i.e., threshold value 0 for $\mathrm{CCW})$ prior to $\mathrm{CW}$ commands. In the generation of $\mathrm{CCW}$ commands, neuron $7 \mathrm{~d}$ exhibited heightened activity $((33.23 \pm 1.16) \mathrm{Hz}$, i.e., threshold value 3 for $\mathrm{CCW})$, while unit $7 \mathrm{~b}$ exhibited a lowered firing rate $((17.91 \pm 1.04) \mathrm{Hz}$, i.e., threshold value 0 for $\mathrm{CW}$ ). STOP commands were generated when activity rates between both neurons were not significantly different (7b $((23.68 \pm 0.72) \mathrm{Hz})$ vs. $7 \mathrm{~d}((21.34 \pm 0.57) \mathrm{Hz}): P>0.05$; Figure 2C). However, corresponding threshold values for $7 \mathrm{~b}$ and $7 \mathrm{~d}$ were $\mathrm{CW} 0$ and CCW 1 , respectively. This appeared to contradict to the triple-threshold comparator algorithm to generate STOP commands. The finding that 7d's activities were not significantly different $(P>0.05)$ for the generation of CCW 1 and CCW 0 suggests that STOP commands mainly occurred when both neurons' activities were at the threshold value 0 . Neuron $7 \mathrm{~b}$ exhibited significantly different firing rates for each of three commands (CW vs. CCW, $P<0.001$; CCW vs. STOP, $P<0.01$; CW vs. STOP, $P<0.001)$ and threshold values segregated into two levels, fluctuating mainly between $\mathrm{CW} 3$ and $\mathrm{CW} 0$. Neuron 7d exhibited substantially different activity between $\mathrm{CW}$ and $\mathrm{CCW}$ rotations $(P<0.001)$ and between $\mathrm{CCW}$ and STOP commands $(P<0.001)$. Threshold values varied among CCW 3, CCW 1 

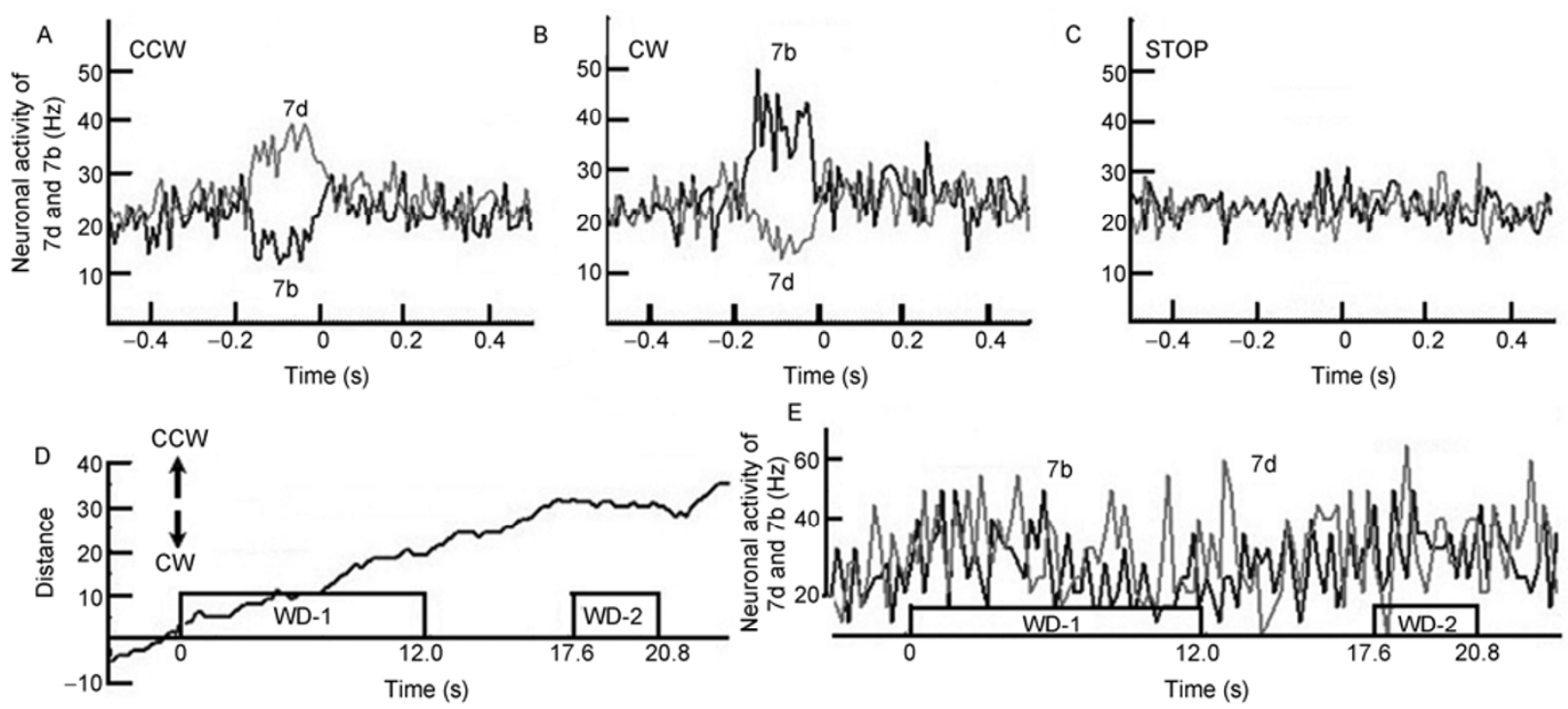

F

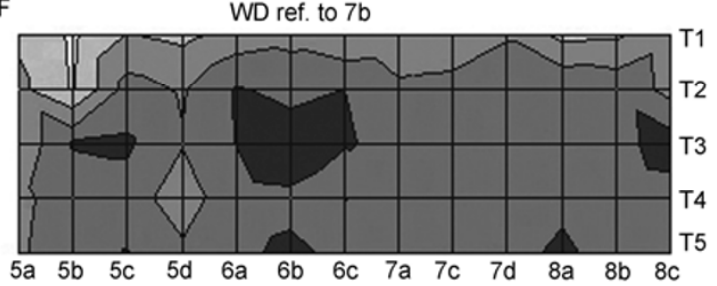

H

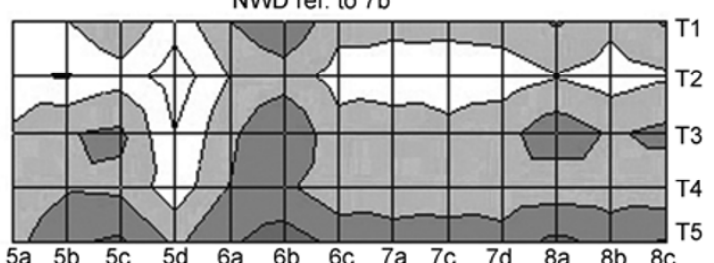

G

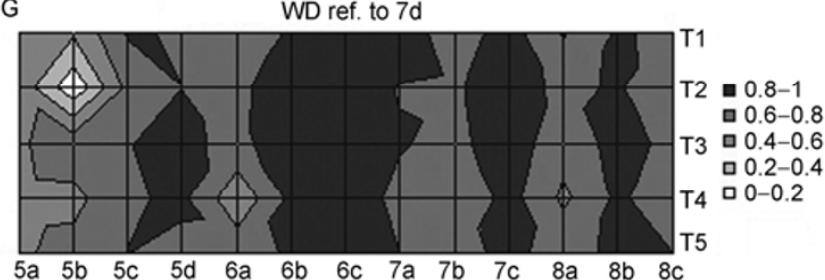

I

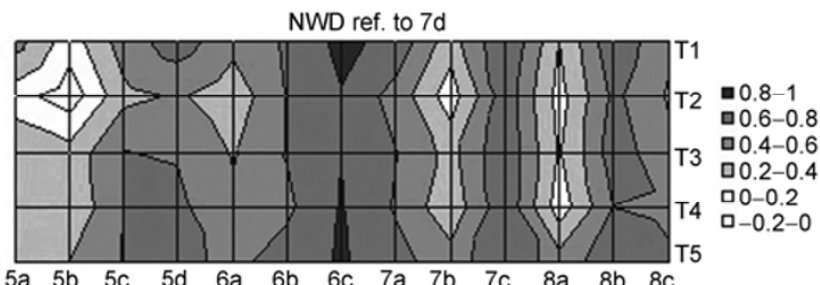

Figure 2 A, Opposite neuronal activity change producing CCW command. B, Opposite neuronal activity change producing CW command. C, Neuronal activity generating a STOP command. D, The distance of the dish within two WD durations. E, Two neuronal activities occurring in the same period. F-I, Correlations between other cells and cells $7 \mathrm{~b}$ and $7 \mathrm{~d}$ from T1 to T5.

and/or 0. Thus, CCWs tended to involve three-step movements, while CWs tended to involve either three- or two-step movements. This ensured the dominant generation of $\mathrm{CCW}$ over CW. Similar activity ranges of neuron $7 \mathrm{~d}$ also provided the flexibility to generate either CW 2 or STOP commands. Although for background periods of 301 to 500 $\mathrm{ms}$ prior to and $500 \mathrm{~ms}$ post to $\mathrm{CW}$ command generation neurons $7 \mathrm{~b}((24.35 \pm 0.45) \mathrm{Hz})$ and $7 \mathrm{~d}((24.21 \pm 0.42) \mathrm{Hz})$ showed similar firing rates, $7 \mathrm{~b}$ exhibited significantly lower activities than $7 \mathrm{~d}(7 \mathrm{~b},(22.20 \pm 0.40) \mathrm{Hz} ; 7 \mathrm{~d},(24.39 \pm 0.36)$ $\mathrm{Hz}, P<0.001)$ for corresponding background periods in $\mathrm{CCW}$ command generation. Thus, $7 \mathrm{~b}$ more strictly obeyed the triple-step threshold algorithm than $7 d$ during WD periods of $\mathrm{T} 5$, suggesting a flexible role of $7 \mathrm{~d}$ in generating more STOP commands to maintain water access, and more $\mathrm{CW}$ rotation commands to counteract $\mathrm{CCW}$ movement. During this WD period of T5, overall, STOP commands were the most frequent, while $\mathrm{CW}$ commands were the least frequent (in \%; STOP, 43.33; CCW, 34.83; CW, 21.84).

During NWD periods of T5, activities of neurons $7 \mathrm{~b}$ and $7 \mathrm{~d}$ also showed similar trends for command generation as in the WD periods. However, in contrast to WD periods, both neurons exhibited distinctively different firing rates for each of three commands (in $\mathrm{Hz}$, Neuron 7b: CW, 35.74 \pm 1.45 ; CCW, 18.47 \pm 0.75 ; STOP, 23.93 \pm 0.53 ; CW vs. CCW, $P<0.001$; CCW vs. STOP, $P<0.001$; CW vs. STOP,

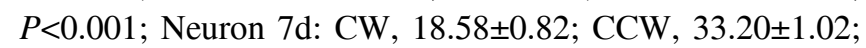
STOP, 22.38 \pm 0.43 ; CW vs. CCW, $P<0.001$; CCW vs. STOP, $P<0.001$; $C W$ vs. STOP, $P<0.05)$. In addition, both neurons also showed significantly different background firing rates for the period of 301 to $500 \mathrm{~ms}$ prior to and 500 $\mathrm{ms}$ after generation of each of three commands (in $\mathrm{Hz}$, Neuron 7b: CW, 25.54 \pm 0.30 ; CCW, 23.53 \pm 0.21 ; STOP,

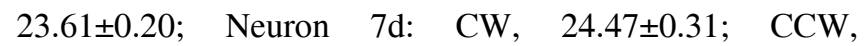
$25.78 \pm 0.22$; STOP, $24.87 \pm 0.23$; $7 \mathrm{~b}$ vs. $7 \mathrm{~d}$ : CW, $P<0.05$; CCW, $P<0.001$; STOP, $P<0.01)$. Thus, during NWD peri- 
ods, both neurons strictly followed the triple-step threshold algorithm more closely than during WD periods of T5. During the NWD period of T5, overall, STOP commands were the most frequent and $\mathrm{CW}$ commands were the least frequent (in \%, STOP, 40.23; CCW, 37.19; CW, 22.58).

During the 6 min of T5, there were 29 WD and 30 NWD periods. Wheel movements in two WD and three NWD periods are shown in Figure 2D. In this example, WD-1 consisted of several repetitions of two different modes of water drinking, i.e., water drinking while issuing $\mathrm{CCW}$ wheel rotations (WD-CCWs) and water drinking during fine control (WD-FCs) of wheel rotations while generating combinations of three commands. During WD-CCWs, the water-containing region of the rotating wheel moved towards the rat at the beginning of the WD-1 period and moved away from the rat at the later period of the WD-1. During WD-FCs, the wheel movements were maintained around a fixed position because of many STOP commands and antagonistic actions between $\mathrm{CW}$ and $\mathrm{CCW}$ commands. In contrast to the WD-1 period, the WD-2 period involved many WD-FCs; the WD-1 period contained $23 \mathrm{CCWs}$, seven CWs and 30 STOPs, while the WD-2 period contained four CCWs, four CWs and 13 STOPs. The relative predominance of CCWs over CWs commands during the WD-1 period is demonstrated by the finding that mean firing rate of $7 \mathrm{~d}((29.79 \pm 1.92) \mathrm{Hz}$, i.e., threshold value 3 for CCW $)$ was significantly $(P=0.0186)$ higher than that of $7 \mathrm{~b}$ $((23.51 \pm 1.79) \mathrm{Hz}$, i.e., threshold value 2 for $\mathrm{CW}$, Figure $2 \mathrm{E})$. During the WD-2 period, the mean activity of $7 \mathrm{~b}$ $((31.47 \pm 2.53) \mathrm{Hz}$, i.e., threshold value 3 for $\mathrm{CW})$ was similar to that of $7 \mathrm{~d}((30.59 \pm 3.45) \mathrm{Hz}$, i.e., threshold value 3 for $\mathrm{CCW}$ ), ensuring more frequent generation of STOP (i.e., CCW 3-CW 3=0) commands.

As indicated above, two units ( $7 \mathrm{~b}$ and $7 \mathrm{~d}$ ) were directly responsible for command generation. Throughout T1-T5, units $7 \mathrm{~b}$ and $7 \mathrm{~d}$ showed strong positive correlations $((r)>0.6)$ during WD periods (Figure $2 \mathrm{~F}$ and $\mathrm{G}$ ), but were not significantly correlated during NWD periods (Figure $2 \mathrm{H}$ and I). Another 12 neurons simultaneously recorded close to $7 \mathrm{~b}$ and $7 \mathrm{~d}$ were also involved, either directly or indirectly, in command generation. These neurons were differentially related to activity changes of either $7 \mathrm{~b}$ or $7 \mathrm{~d}$ during the WD and NWD periods (Figure 2F-I, Table 1). In general, unit 7d (Figure 2G-I) exhibited a higher positive correlations to other neurons compared to unit $7 \mathrm{~b}$ (Figure $2 \mathrm{~F}-\mathrm{H}$ ), regardless of WD or NWD period. Thus, these results suggest that highly correlated influences from other neurons might be required for the flexible role of unit $7 \mathrm{~b}$ in generating appropriate commands to enable water access during WD periods. Eight units $(5 \mathrm{c}, 5 \mathrm{~d}, 6 \mathrm{~b}, 6 \mathrm{c}, 7 \mathrm{a}, 7 \mathrm{c}, 8 \mathrm{~b}$, and $8 \mathrm{c}$ ) showed strong correlations $((r)>0.6)$ with unit $7 \mathrm{~d}$ during WD periods throughout five trials, but only three units $(6 \mathrm{c}, 7 \mathrm{c}$, and $8 b$ ) exhibited strong correlations during NWD periods (Table 1). None of the 12 units showed any correlation with unit $7 \mathrm{~b}$ during WD period of $\mathrm{T} 1$, but eight units $(5 \mathrm{c}, 6 \mathrm{a}, 6 \mathrm{~b}$, $6 \mathrm{c}, 7 \mathrm{a}, 7 \mathrm{c}, 8 \mathrm{a}$, and $8 \mathrm{~b}$ ) exhibited strong relations during the WD period of T2. During the WD period of T3 and T5, all units except unit 5a exhibited strong correlations with $7 \mathrm{~b}$. During T4, all units, except 5a and 5d, exhibited strong correlations with unit $7 \mathrm{~b}$. Thus, from $\mathrm{T} 2$ to $\mathrm{T} 5$, six units $(5 \mathrm{c}$, $6 \mathrm{~b}, 6 \mathrm{c}, 7 \mathrm{a}, 7 \mathrm{c}$, and $8 \mathrm{~b}$ ) exhibited strong correlations with both $7 \mathrm{~b}$ and $7 \mathrm{~d}$ during WD periods. However, unit 5a was not correlated to either neuron regardless of WD or NWD period. Units $5 \mathrm{~d}$ and $8 \mathrm{c}$ exhibited more preferential correlations with unit 7d than unit 7b during WD periods of T1-T5, whereas units $5 \mathrm{~b}, 6 \mathrm{a}$, and $8 \mathrm{a}$ showed a preference for unit $7 \mathrm{~b}$ over unit $7 \mathrm{~d}$. Thus, units $5 \mathrm{~d}$ and $8 \mathrm{c}$ might preferentially modulate unit $7 \mathrm{~d}$ 's activity in generating threshold values of either $\mathrm{CCW} 0$ or $\mathrm{CCW} 1$. In addition, units $5 \mathrm{~b}, 6 \mathrm{a}$, and $8 \mathrm{a}$ might be preferentially involved in flexible control of unit $7 \mathrm{~b}$ 's activity to generate threshold values of either CW 0 or CW 3. However, these preferential influences on $7 \mathrm{~b}$ and $7 \mathrm{~d}$ appeared to be indirect, since cross-correlation analyses did not reveal any immediate (presumably synaptic) modulations. During NWD periods throughout T1-T5, unit 7b was not correlated with most other simultaneously recorded units, except for three units $(5 c, 6 b$, and $8 a)$ at T5. Three other units $(5 \mathrm{a}, 5 \mathrm{~b}$, and $6 \mathrm{a})$ were related to neither $7 \mathrm{~b}$ nor $7 \mathrm{~d}$ units during NWD periods throughout T1-T5.

Other simultaneously recorded neurons' activities at T5 were also analyzed to test whether they had any correlation with either unit $7 \mathrm{~b}$ or $7 \mathrm{~d}$ during the $200 \mathrm{~ms}$ prior to each command generation (Table 2). During the overall WD period of $\mathrm{T} 5$, neither $7 \mathrm{~b}$ nor $7 \mathrm{~d}$ exhibited a significant correlation with other simultaneously recorded neurons. However, $7 \mathrm{~b}$ and $7 \mathrm{~d}$ exhibited negative relation $((r)=-0.61)$ to each other prior to $\mathrm{CW}$ command generation and mild relation (but significant, $(r)=-0.54)$ between them prior to $\mathrm{CCW}$ command generation during WD periods of T5. During NWD periods of $\mathrm{T} 5$, much stronger negative correlations between two units were found prior to $\mathrm{CCW}$ generation $((r)=-0.88)$ than prior to $\mathrm{CW}$ output $((r)=-0.62)$. Unit $6 \mathrm{c}$ exhibited a strong positive correlation with unit $7 \mathrm{~d}$, and a negative correlation with unit $7 \mathrm{~b}$ during the NWD period. These correlation analyses coincided with $\mathrm{CCW}$ dominance (i.e., threshold value 3 for $\mathrm{CCW}$ ) during the NWD period and the importance of CW movements (i.e., threshold value 3 for $\mathrm{CW}$ ) during the WD period. Units $7 \mathrm{~b}$ and $7 \mathrm{~d}$ did not exhibit any significant correlations between them prior to generating STOP commands during both WD $((r)=-0.1076)$ and NWD $((r)=-0.0414)$ periods of T5. This was natural, since the threshold value for $\mathrm{CW} 0$ was generated consistently by the different mean firing rates of $7 b$ during the WD and NWD periods. Threshold values for CW 0 and CW 1 were determined by the lack of a significant difference between 7d's mean firing rates responsible for the two commands (as stated earlier) during the WD period.

The results indicated that a trial-dependent increase of 
Table 1 Correlations between control signals in $7 \mathrm{~b}$ and $7 \mathrm{~d}$ and signals in other cells ${ }^{\mathrm{a})}$

\begin{tabular}{|c|c|c|c|c|c|c|c|c|c|c|c|c|c|c|}
\hline Ref & $7 \mathrm{~d}$ & $5 \mathrm{a}$ & $5 b$ & $5 \mathrm{c}$ & $5 \mathrm{~d}$ & $6 a$ & $6 \mathrm{~b}$ & $6 c$ & $7 \mathrm{a}$ & $7 b$ & $7 \mathrm{c}$ & $8 \mathrm{a}$ & $8 \mathrm{~b}$ & $8 \mathrm{c}$ \\
\hline \multirow{5}{*}{ WD } & T5 & 0.57 & $0.67 *$ & $0.80 *$ & $0.74 *$ & $0.74 *$ & $0.82 *$ & $0.89 *$ & $0.80 *$ & $0.75 *$ & $0.84 *$ & $0.74 *$ & $0.83 *$ & $0.80^{*}$ \\
\hline & $\mathrm{T} 4$ & 0.53 & 0.54 & $0.77 *$ & $0.84 *$ & 0.45 & $0.84 *$ & $0.92 *$ & $0.75 *$ & $0.61 *$ & $0.86^{*}$ & 0.56 & $0.83^{*}$ & $0.68^{*}$ \\
\hline & $\mathrm{T} 3$ & 0.57 & $0.73 *$ & $0.80 *$ & $0.83 *$ & $0.76^{*}$ & $0.89 *$ & $0.93 *$ & $0.82 *$ & $0.74 *$ & $0.91 *$ & $0.67 *$ & $0.88 *$ & $0.74 *$ \\
\hline & $\mathrm{T} 2$ & 0.53 & 0.05 & $0.65^{*}$ & $0.80 *$ & $0.75^{*}$ & $0.94 *$ & $0.90 *$ & $0.79 *$ & $0.77 *$ & $0.89 *$ & $0.75^{*}$ & $0.82 *$ & $0.60 *$ \\
\hline & $\mathrm{T} 1$ & 0.57 & 0.43 & $0.82 *$ & $0.78 *$ & $0.66^{*}$ & $0.82 *$ & $0.87 *$ & $0.90 *$ & $0.70 *$ & $0.88 *$ & 0.58 & $0.82 *$ & $0.76^{*}$ \\
\hline Ref & $7 \mathrm{~b}$ & $5 a$ & $5 b$ & $5 c$ & $5 \mathrm{~d}$ & $6 a$ & $6 \mathrm{~b}$ & $6 \mathrm{c}$ & $7 \mathrm{a}$ & $7 \mathrm{~b}$ & $7 \mathrm{~d}$ & $8 \mathrm{a}$ & $8 \mathrm{~b}$ & $8 \mathrm{c}$ \\
\hline \multirow{5}{*}{ WD } & T5 & 0.57 & $0.71 *$ & $0.81^{*}$ & $0.65 *$ & $0.78^{*}$ & $0.82 *$ & $0.77 *$ & $0.76^{*}$ & $0.76^{*}$ & $0.75 *$ & $0.83 *$ & $0.74^{*}$ & $0.76^{*}$ \\
\hline & $\mathrm{T} 4$ & 0.55 & $0.72 *$ & $0.71 *$ & 0.48 & $0.76^{*}$ & $0.77 *$ & $0.60 *$ & $0.64 *$ & $0.60 *$ & $0.61 *$ & $0.77^{*}$ & $0.68 *$ & $0.70^{*}$ \\
\hline & $\mathrm{T} 3$ & 0.43 & $0.81 *$ & 0.83 & $0.61^{*}$ & $0.80 *$ & $0.89 *$ & $0.82 *$ & $0.71 *$ & $0.76 *$ & $0.74 *$ & $0.72 *$ & $0.72 *$ & $0.90^{*}$ \\
\hline & $\mathrm{T} 2$ & 0.45 & 0.17 & $0.68 *$ & 0.58 & $0.81 *$ & 0.75 & $0.80 *$ & $0.64 *$ & $0.67 *$ & $0.77 *$ & $0.78^{*}$ & $0.74 *$ & 0.53 \\
\hline & $\mathrm{T} 1$ & 0.37 & 0.16 & 0.41 & 0.35 & 0.47 & 0.52 & 0.42 & 0.44 & 0.48 & 0.57 & 0.34 & 0.37 & 0.52 \\
\hline Ref & $7 \mathrm{~d}$ & $5 \mathrm{a}$ & $5 b$ & $5 c$ & $5 \mathrm{~d}$ & $6 a$ & $6 \mathrm{~b}$ & $6 \mathrm{c}$ & $7 \mathrm{a}$ & $7 \mathrm{~b}$ & $7 \mathrm{c}$ & $8 \mathrm{a}$ & $8 \mathrm{~b}$ & $8 \mathrm{c}$ \\
\hline \multirow{5}{*}{ NWD } & T5 & 0.27 & 0.40 & $0.61^{*}$ & $0.65 *$ & 0.56 & $0.68 *$ & $0.82 *$ & $0.66^{*}$ & 0.49 & $0.77 *$ & 0.50 & $0.72 *$ & $0.63^{*}$ \\
\hline & $\mathrm{T} 4$ & 0.23 & 0.22 & $0.60 *$ & $0.64 *$ & 0.44 & 0.57 & $0.81 *$ & 0.58 & 0.26 & $0.76^{*}$ & 0.09 & $0.60 *$ & 0.56 \\
\hline & $\mathrm{T} 3$ & 0.39 & 0.27 & $0.60 *$ & 0.59 & 0.38 & $0.61 *$ & $0.80 *$ & $0.61 *$ & 0.33 & $0.75^{*}$ & 0.17 & $0.71 *$ & 0.45 \\
\hline & $\mathrm{T} 2$ & 0.07 & -0.12 & 0.34 & 0.42 & 0.27 & $0.60 *$ & $0.79 *$ & 0.52 & 0.09 & $0.72 *$ & 0.12 & $0.65^{*}$ & 0.38 \\
\hline & $\mathrm{T} 1$ & 0.49 & 0.09 & 0.50 & $0.71 *$ & 0.46 & $0.62 *$ & $0.85 *$ & $0.74 *$ & 0.29 & $0.80 *$ & 0.20 & $0.75^{*}$ & 0.44 \\
\hline Ref & $7 \mathrm{~b}$ & $5 \mathrm{a}$ & $5 b$ & $5 c$ & $5 d$ & $6 a$ & $6 \mathrm{~b}$ & $6 c$ & $7 \mathrm{a}$ & $7 b$ & $7 \mathrm{~d}$ & $8 \mathrm{a}$ & $8 \mathrm{~b}$ & $8 \mathrm{c}$ \\
\hline \multirow{5}{*}{ NWD } & T5 & 0.34 & 0.57 & $0.63 *$ & 0.36 & 0.58 & $0.70 *$ & 0.55 & 0.50 & 0.54 & 0.49 & $0.65^{*}$ & 0.54 & $0.62 *$ \\
\hline & $\mathrm{T} 4$ & 0.20 & 0.38 & 0.36 & 0.08 & 0.41 & 0.44 & 0.25 & 0.27 & 0.25 & 0.26 & 0.28 & 0.28 & 0.26 \\
\hline & $\mathrm{T} 3$ & 0.24 & 0.39 & 0.43 & 0.00 & 0.36 & 0.56 & 0.28 & 0.33 & 0.26 & 0.33 & 0.52 & 0.37 & 0.45 \\
\hline & $\mathrm{T} 2$ & 0.01 & -0.01 & 0.12 & -0.12 & 0.21 & 0.32 & 0.12 & 0.07 & 0.08 & 0.09 & 0.19 & 0.09 & 0.17 \\
\hline & $\mathrm{T} 1$ & 0.20 & 0.19 & 0.37 & 0.09 & 0.40 & 0.55 & 0.28 & 0.28 & 0.26 & 0.29 & 0.42 & 0.26 & 0.42 \\
\hline
\end{tabular}

a) $*, P<0.05$.

Table 2 Neuronal activities $200 \mathrm{~ms}$ prior to STOP, CCW, and CW commands were correlated to Ref 7b and 7d during WD and NWD ${ }^{\text {a) }}$

\begin{tabular}{|c|c|c|c|c|c|c|c|c|c|c|c|c|c|c|c|}
\hline & WD & $5 \mathrm{a}$ & $5 b$ & $5 c$ & $5 d$ & $6 a$ & $6 b$ & $6 \mathrm{c}$ & $7 \mathrm{a}$ & $7 \mathrm{~b}$ & $7 \mathrm{c}$ & $7 d$ & $8 \mathrm{a}$ & $8 b$ & $8 \mathrm{c}$ \\
\hline \multirow{2}{*}{ STOP } & Ref $7 b$ & -0.11 & 0.29 & -0.14 & -0.12 & 0.10 & 0.30 & -0.27 & 0.20 & & -0.30 & -0.11 & -0.07 & 0.02 & -0.06 \\
\hline & $\operatorname{Ref} 7 d$ & -0.44 & -0.22 & 0.23 & -0.27 & -0.07 & -0.12 & -0.44 & -0.48 & -0.11 & -0.24 & & 0.06 & -0.14 & 0.16 \\
\hline \multirow{2}{*}{$\mathrm{CCW}$} & $\operatorname{Ref} 7 b$ & 0.22 & 0.19 & 0.16 & 0.01 & 0.18 & -0.28 & -0.10 & -0.24 & & 0.00 & $-0.54 *$ & 0.24 & -0.41 & -0.24 \\
\hline & Ref 7d & -0.14 & 0.05 & 0.25 & 0.45 & -0.07 & 0.31 & 0.30 & 0.00 & $-0.54^{*}$ & 0.11 & & -0.01 & 0.34 & 0.49 \\
\hline \multirow{2}{*}{$\mathrm{CW}$} & Ref $7 b$ & 0.31 & 0.27 & 0.32 & 0.22 & 0.09 & 0.33 & -0.01 & 0.15 & & -0.13 & $-0.61 *$ & 0.30 & 0.32 & 0.22 \\
\hline & Ref 7d & -0.30 & -0.12 & -0.31 & -0.28 & -0.39 & -0.01 & 0.28 & -0.24 & $-0.61 *$ & -0.14 & & -0.17 & -0.04 & -0.36 \\
\hline \multirow{2}{*}{ STOP } & Ref $7 b$ & -0.32 & 0.12 & -0.05 & 0.30 & 0.15 & 0.36 & 0.20 & -0.18 & & 0.05 & -0.04 & 0.02 & 0.40 & -0.21 \\
\hline & $\operatorname{Ref} 7 d$ & -0.21 & -0.05 & 0.06 & -0.24 & -0.16 & 0.41 & 0.07 & -0.25 & -0.04 & -0.02 & & 0.23 & -0.25 & 0.12 \\
\hline \multirow{2}{*}{$\mathrm{CCW}$} & $\operatorname{Ref} 7 b$ & -0.21 & 0.19 & -0.29 & -0.33 & 0.12 & -0.33 & $-0.64 *$ & 0.21 & & -0.51 & $-0.88 *$ & -0.09 & -0.31 & -0.05 \\
\hline & $\operatorname{Ref} 7 d$ & 0.04 & -0.22 & 0.36 & 0.39 & -0.09 & 0.32 & $0.64 *$ & -0.11 & $-0.88^{*}$ & 0.25 & & 0.14 & 0.43 & -0.06 \\
\hline \multirow{2}{*}{$\mathrm{CW}$} & Ref $7 b$ & -0.07 & 0.33 & -0.13 & -0.07 & 0.20 & 0.21 & -0.08 & 0.29 & & -0.10 & $-0.62 *$ & 0.40 & -0.07 & 0.29 \\
\hline & Ref 7d & 0.28 & -0.25 & 0.16 & 0.12 & -0.11 & -0.06 & 0.34 & -0.43 & $-0.62 *$ & -0.02 & & -0.14 & 0.25 & 0.05 \\
\hline
\end{tabular}

a) $*, P<0.05$.

total water drinking duration (TWD duration) (\%) was strongly $((r)=0.9053)$ correlated with the change in WD frequency (Figure 3A). This trial-dependent WD frequency augmentation was predominantly related to the change of CW $((r)=0.9920)$ movement, and average water drinking duration (AWD duration) was highly related to the occurrence of STOP commands $((r)=0.9419$; Figure 3B). If water dish control using the BCI system to quench thirstiness was motivational, the proportion of the generation of the three commands might be different among various behavioral states. During the 6 min sleep period, in which rats did not have any motivation to drink water and presumably no motivation to control the BCI system, CW commands were rare, while STOP commands were numerous (Figure 3C). During WD periods of T5, CW commands were dominant, whereas $\mathrm{CCW}$ were the least prevalent. However, during NWD periods of T5 (T-NWD), CW and CCW were observed in similar proportions. Command proportions during the NWD periods were quite similar to those when water dish control was allowed but water access was blocked by placing the water dish far away from the rat (FAR in Figure $3 \mathrm{C})$. This result might be expected, since rats were moti- 

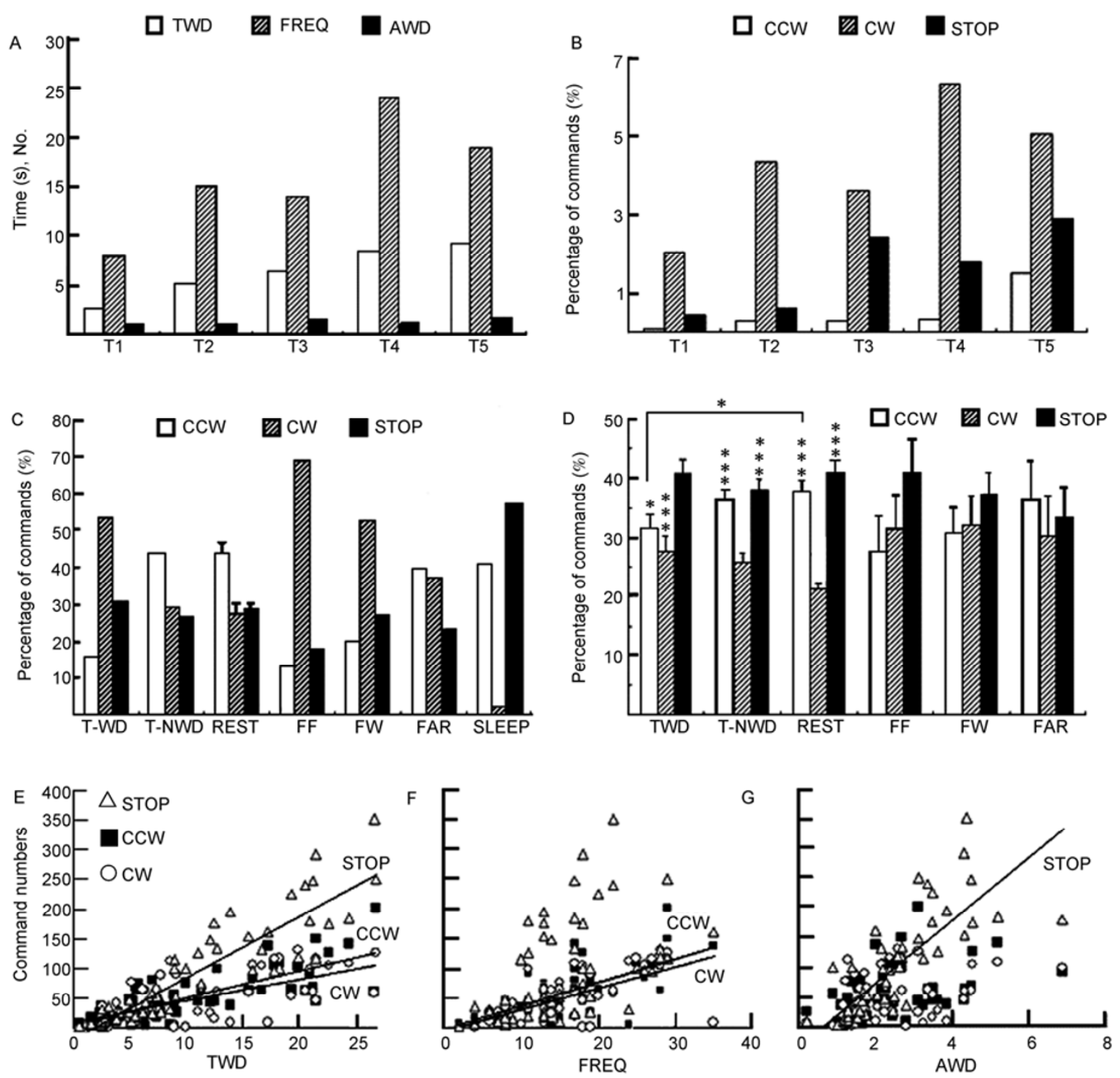

Figure 3 A, Histogram showing total WD, frequency, and AWD in five trials. B, Histogram of CCW, CW, and STOP commands in different trials. C, Histogram of distribution of three commands in different sessions. D, Statistical comparison of CCW, CW, and STOP commands in different sessions of 16 rats. *, $P<0.05$; **, $P<0.01$; ***, $P<0.001$. E-G, Correlations of $\mathrm{CW}, \mathrm{CCW}$, and STOP commands with TWD, frequency, and AWD.

vated to control the BCI system during the T-NWD and FAR periods, but experienced difficulty accessing the water dish. During six repetitions of the resting (REST) periods between five trial periods, rats had to wait $6 \mathrm{~min}$ to start new WD trial since the chamber was blocked by paper, and thereby would be expected to give up the motivation of controlling the BCI system. CCW commands were most frequently observed in this period. Proportions of the three commands generated when the rat was allowed free access to water (FW) by fixing the water dish in front of the rat were similar to those in the T-WD condition. However, the frequencies of $\mathrm{CW}$ and $\mathrm{CCW}$ were different between the two behavioral states. The proportions of the three commands generated when the rat had free access to food (FF; by placing food pellets in the rat chamber) were similar to those in the T-WD condition. However, the frequencies of CCW and STOP commands were different between the two behavioral states. Overall, the proportions of command generation during the T-WD, FF, and $\mathrm{FW}$ periods indicated that CW commands occurred commonly, whereas the T-NWD, REST, and FAR periods were associated with more frequent $\mathrm{CCW}$ commands. Averaged proportions of the generation of the three commands from 16 rats during various behavioral states (T-WD, $n=59$; T-NWD, $n=59$; REST, $n=102$; FF, $n=14$; FW, $n=14$; FAR, $n=4$ ) are shown in Figure 3D. During T-WD, STOP commands were significantly more numerous than either $\mathrm{CW}$ or $\mathrm{CCW}$ commands. However, during both T-NWD and REST periods, CW commands were significantly fewer than either $\mathrm{CCW}$ or STOP commands. In addition, CCW occurrence during 

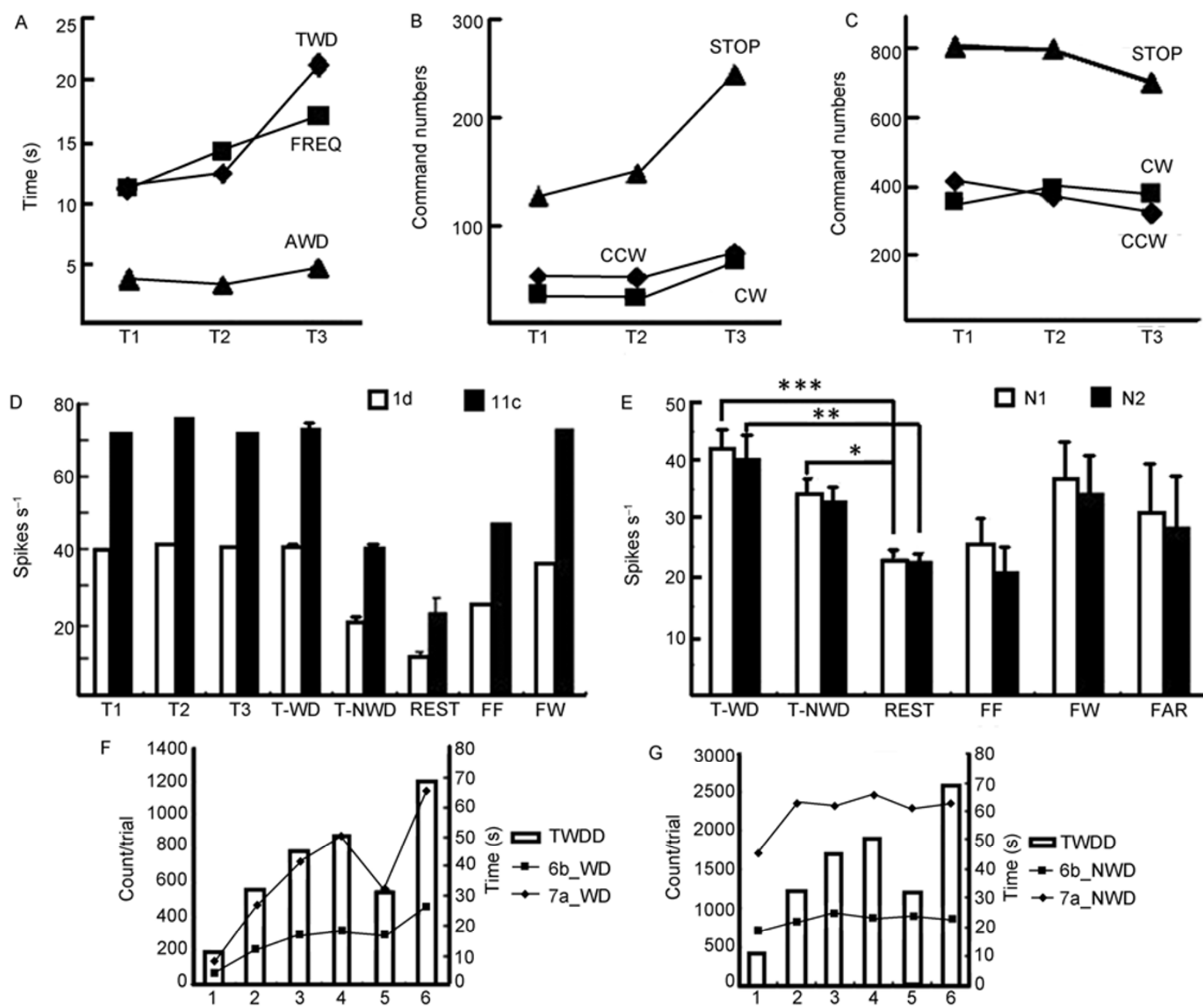

Figure 4 A, TWD, frequency, and AWD changes in the first three trials. B, Change in CCW, CW, and STOP commands in the first three trials during WD. $\mathrm{C}$, Change in $\mathrm{CCW}, \mathrm{CW}$, and STOP commands in the first three trials during NWD. D, Activities of two controlling neurons in different sessions. E, Statistical analysis of neuronal activity in different sessions. *, $P<0.05 ; * *, P<0.01 ; * * *, P<0.001$. F and G, Burst analysis. Maximum interval to start burst, $0.02 \mathrm{~s}$; maximum interval to end burst, $0.02 \mathrm{~s}$; minimum interval between bursts, $0.02 \mathrm{~s}$; minimum duration of burst, $0.08 \mathrm{~s}$; minimum number of spikes in burst, 4 . F, WD period; G, non-WD period.

REST was significantly more frequent than during T-WD. Averaged proportions of three command generations were similar during the FF, FW, and FAR periods. During REST, CW occurrence was significantly less frequent than during either the T-NWD or FW periods. The relationships between BCI efficiency (TWD, FREQ, and AWD) and three commands generated during T-WDs from 56 trials in 16 rats were analyzed (Figure $3 \mathrm{E}-\mathrm{G}$ ). TWD changes from trial to trial exhibited linear correlations with all three commands (CCW, $(r)=0.7696 ; \mathrm{CW}, \quad(r)=0.6596)$, and were most strongly correlated with the STOP command $((r)=0.8980)$. WD frequency was linearly related to both $\mathrm{CCW}$ $((r)=0.6982)$ and CW $((r)=0.6732)$ commands. AWD duration was significantly correlated with the STOP command $((r)=0.7922)$.

The experimental results revealed that TWD (\%) and WD frequency were increased from T1 to T3 (Figure 4A).
During T-WD periods, the frequency of STOP $((r)=0.9970)$ commands was related to TWD changes (Figure 4B). At T3, the frequency of all three commands was increased. During T-NWD periods, both STOP and CCW commands decreased in frequency from $\mathrm{T} 1$ to $\mathrm{T} 3$. In addition, neurons $1 \mathrm{~d}$ and $11 \mathrm{c}$ were set to encode $\mathrm{CCW}$ and $\mathrm{CW}$ commands, respectively (Figure 3D). Triple-step thresholds for unit 1d were set to $30,20,10$ in $\mathrm{Hz}$ (CCW threshold values: above 30: 3, 20-29: 2, 10-19: 1, below 19: 0) and those of 11c were to 60,40 , and 20 in $\mathrm{Hz}$ (CW threshold values: above 40: 3, 40-39: 2, 20-39: 1, below 20: 0). Firing rates of both $1 \mathrm{~d}$ and $11 \mathrm{c}$ were above each neuron's threshold level 3 during T-WD periods from T1 to T3. During T-NWD periods, the activities of both $1 \mathrm{~d}$ and $11 \mathrm{c}$ were a range that could generate each neuron's threshold value of either 2 or 1 . During resting periods, both neurons' firings were at levels for generating threshold values of either 1 or 0 . Average 

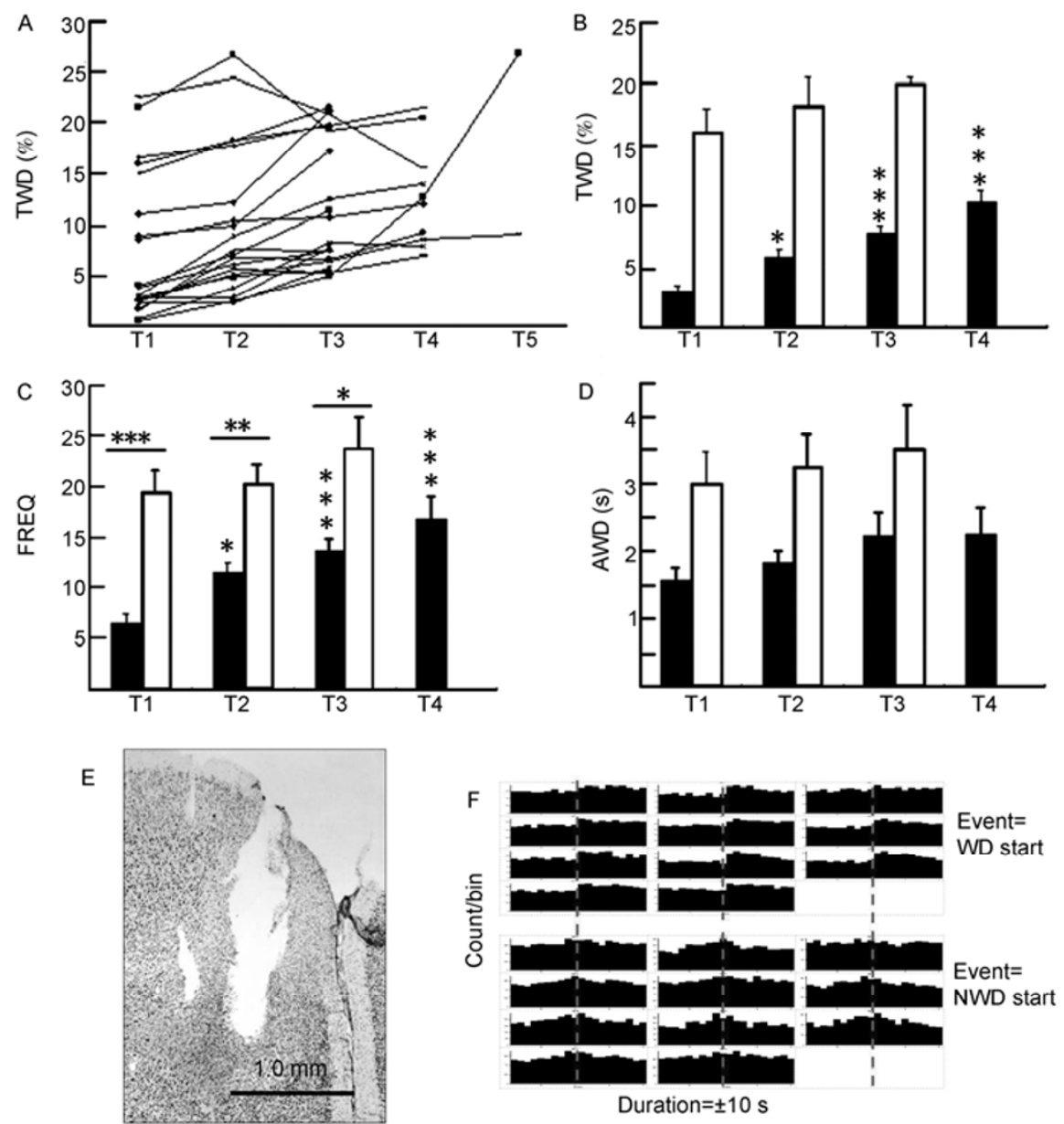

Figure 5 A, Percentage of TWD in all experiments. B-D, Change of TWD, frequency, and AWD in all experiments in the first four trials. *, $P<0.05$; **, $P<0.01$; ***, $P<0.001$. E, Histology of implantation site in the PFC (cresyl violet stain). F, Perievent histogram of 11 signals. The dotted line shows the initial of WD (above) and NWD (below).

activity of $1 \mathrm{~d}$ during rest was significantly lower than during T-NWD $(P=0.0024)$ and during T-WD $(P=0.0238)$. Average activity of $11 \mathrm{c}$ during rest was also significantly lower from that during T-NWD $(P=0.0238)$ and that during T-WD $(P<0.0001)$. Activity of $1 \mathrm{~d}$ and $11 \mathrm{c}$ during FW period was similar to that during the T-WD period. Activity of both neurons during FF was similar to those during the T-NWD period. Averaged activities of N1s and N2s from 16 rats during the resting period were significantly lower than during either T-WD or T-NWD (Figure 4E). In another case, the number of firing bursts exhibited by neuron $6 \mathrm{~b}$ and $7 \mathrm{a}$ in WD and NWD was compared in Figure 4F and G. A significant correlation $(P=0.0018$ for $6 \mathrm{~b}, P<0.0001$ for $7 \mathrm{a})$ was found between neuronal bursting and duration of WD, but this was not found for NWD $(P=0.2125$ for $6 \mathrm{~b}$, $P=0.0782$ for $7 \mathrm{a})$.

In Figure 5, trial-dependent BCI efficiency changes obtained from 16 rats were examined in terms of \% TWD duration, frequency of WD and AWD duration per trial. TWD $(\%)$ at $\mathrm{T} 1$ was found to be variable, ranging from $0.56 \%$ to $21.54 \%$ in the 6 min period (Figure 5A). Thirteen of 20 rats showed trial-dependent gradual increases of BCI efficiency measured by TWD and WD frequency (Figure 5B and C). Most of these rats showed less than 5\% TWD at T1. The seven rats not showing trial-dependent BCI efficiency increases tended to exhibit higher TWD (8.96\%-22.67\%), even at T1. Averaged WD durations per trial did not change as trials were repeated. Thus, BCI efficiency augmentation occurred when initial efficiency was quite low. This mainly occurred with the increment of WD frequency. The WD amount was measured after each trial, and averaged WD amount per second was $(0.049 \pm 0.010) \mathrm{mL}$. Figure $5 \mathrm{~F}$ shows neuronal activity pre- and post-WD, with a bin size of $1 \mathrm{~s}$. Neuronal activity significantly increased in WD compared to pre-WD $(P<0.01)$, and post-WD neuron activity gradually decreased in the $10 \mathrm{~s}$ period after WD.

\section{Discussion}

Teuber [25] hypothesized that PFC neurons play a vital role in processing signals from sensory to motor information. 
Here, we found that PFC neurons could function as an adaptive goal-encoding region to fulfill an animal's requirements when spontaneous signals were transformed in real-time through a simple BCI system into commands for controlling a machine. The current study produced the following major findings: (i) Using a simple algorithm, we found that rats were able to control a BCI system using the activity of single neurons in PFC. (ii) PFC neurons provided a feasible adaptive choice for animals to manipulate a machine after learning in several training sessions. (iii) Motivational control of our BCI system was evidenced by differential changes of neuronal activity with experience, and the appropriate generation of three commands during various behavioral states, such as resting, free food, free water, and FAR periods.

A closed loop information stream was constructed in the design of a BCI system: This loop involved a thirsty rat with a motivation to drink, a triple-threshold algorithm (Figure 1) to convert neural signals into machine control commands, and a command execution system. This loop was completed when the rat began to notice and understand the function of the reward system. To investigate whether rats could be trained (i.e., could encode the necessary information) to comprehend the relation between their mental state and the movement of the dish, they were required to accomplish the task of controlling a water-provision system that rotated in 1-D. A trial-dependent BCI efficiency increase was observed. This increase was particularly apparent when initial efficiency at trial 1 was very low (Figure 5). However, when initial efficiency was high above $10 \%$ of 6 min, rats tended to show less motivation to control the BCI system after three trials. Intentional differences were also expressed in the proportions of the three generated commands. In the sleep period there was presumably no motivation to drink water, and thereby no motivation to control the BCI system. The proportions of three commands generated during this sleep period were markedly different from those during WD trial periods (Figure 3C). During the 6 min resting periods between the trial periods, the rat had to wait for the next trial, holding the motivation of WD. Although this is a short interval, the rat also had to wait for the next T-WD period during the T-NWD periods between the T-WD periods. This similarity was reflected in the proportions of three commands between the T-NWD and the resting periods (Figure $3 \mathrm{C}$ and $\mathrm{D}$ ). For trained rats, the rotating dish served as a visual cue. The NWD periods effectively constituted 'delay' durations in the task. This might explain the persistence of neuronal activity that resulted in different proportions of commands from the resting and FAR sessions [26]. During the FAR period, the rat was able to watch the water dish movement with the motivation to drink water, but was unable to access the water for $6 \mathrm{~min}$. Command generation during FW was largely similar to those in T-WD, even though there was no actual control of the water dish movement during this period. Command generation during FF
(Figure 3C) was similar to that during $\mathrm{FW}$, but $\mathrm{CW}$ was further increased while CCW and STOP commands were decreased. This similarity may reflect the common motivation to fulfill its appetite, while the difference may be related to the absence of motivational control of the water dish, since food pellets were given freely in the chamber, rather than on the controllable water dish. The proportion of the three commands generated during the FF, FW, FAR periods was quite variable among 16 rats, but did not significantly differ between rats (Figure 3D). This suggests that the necessity of strict regulation of command generation was commonly absent, compared to during WD trials.

In the current study, maximum BCI efficiency was lower than $30 \%$, and was increased by the elevation of WD frequency. If we suppose that the system was rotated in one direction with a specified angle for a trial without a STOP command, the rat was able to access the water dish for $86^{\circ}$. In this case, the efficiency would be $23.9 \%$, and one cycle of rotation took $3.35 \mathrm{~s}$ for three steps $\left(21.5^{\circ}\right)$, and $4.97 \mathrm{~s}$ for one step $\left(14.5^{\circ}\right)$ of angular movement. Thus, the estimated averaged WD duration ranged from 0.80 to $1.19 \mathrm{~s}$, and WD frequency ranged from 72.5 to 107.5 times for 6 min of a trial period. The overall averaged WD duration was $(2.37 \pm 0.17) \mathrm{s}$ for whole trials $(n=56)$, and the averaged WD frequency was $(15.09 \pm 0.99)$ times for a trial period (the highest WD frequency was 35). These findings indicate that actual BCI control was not performed by simple rotation in one direction (Figure 3F), and that water drinking was not performed by chance during wheel rotation. The occurrence of STOP commands during the fine control period aided the increase in average WD duration (Figure 3G), but most strongly decreased the WD frequency during the non-WD period of a trial. One of the counter actions for the two directional commands was appropriate in the fine control period and for the WD frequency increase (Figure 3F). However, dominant command generation for one direction during the non-WD period served to speed up the next period of access to water (Figure 3D). The lowest performance of the current BCI system could be achieved by fixing or moving the water dish to a position that was not accessible by the rat. This non-accessible region covers $72.1 \%$ of the wheel rotation. On the other hand, the best performance in our BCI system could be achieved by moving the water containing region, while issuing a 1:1 ratio of $\mathrm{CW}$ and CCW commands, and many STOP commands (Figure 3G), directly in front of the rat; the water-containing region covered $23.9 \%$ of the wheel rotation. This type of optimal performance is shown as WD-FC during the WD-2 period in Figure 2D.

Compared with decoding algorithms, encoding algorithms require fewer signals (minimum of two), to reach high efficiency, which in return could perform a greater number of functions using a similar number of neurons in decoding. Accordingly, this algorithm causes less damage than methods requiring massive implantation of brain tissue. 
The remarkable cortical plasticity of the brain means that signals from implanted electrodes can, after adaptation, be utilized by electrodes and computers [27]. Because PFC neurons perform various functions, such as prediction, working memory and discrimination, the mean, maximum, and minimum frequency of PFC neurons in a rat, or among different rats, was not consistent. The average spontaneous

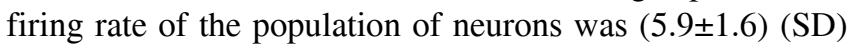
spikes $\mathrm{s}^{-1}$. Although each neuron's absolute activity level was important for the generation of a normalized threshold value, the difference between two normalized threshold values (N1 and N2) encoded from two neurons' activities was the factor determining which of the three commands was given (CW: $3,2,1$; CCW: $-3,-2,-1$; STOP: 0). This algorithm meant that two neurons could fire in a negatively correlated fashion, even though the two neurons were recorded from a single wire, for the generation of $\mathrm{CW}$ and CCW commands, and in an uncorrelated fashion for the STOP command during the $200 \mathrm{~ms}$ pre-command period (Figure 2A and B, Table 2). The effectiveness of this triple threshold comparator algorithm was predominantly restricted to the designated N1 and N2 neurons, although there were many neurons in the vicinity of the two neurons (Table 2). This result may partially reflect that the PFC is not construed simply as a cluster of neurons, but as a network [28].

The trial-dependent BCI efficiency increase was largely due to the increase in WD frequency (Figure 5C), since average WD duration was not significantly altered over subsequent trials (Figure 5D). However, the activities of N1 and N2 during the resting period, when the rat sat in the chamber for 6 min without seeing the water dish (i.e., without the visual cue) and therefore with no motivation to control the BCI system, were significantly lower than those during the T-WD trial period (Figure 4D and E) [29]. The comparison of the number of neuronal bursts in WD with NWD further supports this notion (Figure 4F and G). A number of previous studies have reported that neuronal units exhibit accelerating discharge during delay periods while animals prepare for movement in a variety of tasks, including delay tasks [28,30-33]. In the current study, neuronal activity during T-NWD was intermediate between T-WD and resting periods. Both neurons exhibited elevated activity during motivational control of the water dish, and suppressed activity during resting periods. This finding indicates that STOP commands observed during T-WD period and the resting period were generated by distinct levels of similar threshold values dictated by two neurons.

This work was supported by the Brain Research Center of the 21st Century Frontier Research Program (Grant No. 2009K001280) funded by the Ministry of Education, Science and Technology, Republic of Korea, and the Industrial Source Technology Development Program (Grant No. 10033634-2009-11) of the Ministry of Knowledge Economy (MKE) of Korea, as well as the Priority Research Centers Program through the
National Research Foundation of Korea (NRF) funded by the Ministry of Education, Science and Technology (Grant No. 2009-0094073).

1 Lucas J W, Schiller J S, Benson V. Summary health statistics for U.S. adults: national health interview survey. Vital Health Stat, 2004, 10: 60-62

2 Hochberg L R, Serruya M D, Friehs G M, et al. Neuronal ensemble control of prosthetic devices by a human with tetraplegia. Nature, 2006, 442: 164-171

3 Felton E A, Garell P C, Williams J C, et al. Electrocorticographically controlled brain-computer interfaces using motor and sensory imagery in patients with temporary subdural electrode implants. Report of four cases. J Neurosurg, 2007, 106: 495-500

4 Fabiani G E, McFarland D J, Pfurtscheller G, et al. Conversion of EEG activity into cursor movement by a brain-computer interface (BCI). IEEE Trans Neural Syst Rehabil Eng, 2005, 12: 331-339

5 Cincotti F, Mattia D, Aloise F, et al. Non-invasive brain-computer interface system: towards its application as assistive technology. Brain Res Bull, 2008, 75: 796-803

6 Bai O, Lin P, Vorbach S, et al. A high performance sensorimotor beta rhythm-based brain-computer interface associated with human natural motor behavior. J Neural Eng, 2008, 5: 24-35

7 Velliste M, Perel S, Spalding M C, et al. Cortical control of a prosthetic arm for self-feeding. Nature, 2008, 453: 1098-1101

8 Moritz C T, Perlmutter S I, Fetz E E. Direct control of paralysed muscles by cortical neurons. Nature, 2008, 456: 639-642

9 Kennedy P R, Andreasen D, Ehirim P, et al. Using human extra-cortical local field potentials to control a switch. J Neural Eng, 2004, 1: 72-79

10 Serruya M D, Hatsopoulos N G, Paninski L, et al. Instant neural control of a movement signal. Nature, 2002, 416: 141-142

11 Taylor D M, Tillery S I, Schwartz A B. Direct cortical control of 3d neuroprosthetic devices. Science, 2002, 296: 1829-1832

12 Carmena J M, Lebedev M A, Crist R E. Learning to control a brain-machine interface for reaching and grasping by primates. PLoS Biol, 2003, 1: e42

13 Shenoy K V, Meeker D, Cao S, et al. Neural prosthetic control signals from plan activity. Neuroreport, 2003, 14: 591-596

14 Musallam S, Corneil B D, Greger B, et al. Cognitive control signals for neural prosthetics. Science, 2004, 305: 258-262

15 Olson B P, Si J, Hu J, et al. Closed-loop cortical control of direction using support vector machines. IEEE Trans Neural Syst Rehabil Eng, 2005, 13: 72-80

16 Wessberg J, Stambaugh C R, Kralik J D, et al. Real-time prediction of hand trajectory by ensembles of cortical neurons in primates. Nature, 2000, 408: 361-365

17 Carmena J M, Lebedev M A, Crist R E, et al. Learning to control a brain-machine interface for reaching and grasping by primates. PLoS Biol, 2003, 1: 193-208

18 Serruya M D, Hatsopoulos N G, Paninski L, et al. Instant neural control of a movement signal. Nature, 2002, 416: 141-142

19 Fuster J M. The Prefrontal Cortex, 2nd ed. New York: Raven Press, 1989

20 Salzman C D, Fusi S. Emotion, cognition, and mental state representation in amygdala and prefrontal cortex. Annu Rev Neurosci, 2010, 33: $173-202$

21 Paxinos G, Watson C. The Rat Brain in Stererotaxic coordinates, 4th ed. San Diego: Academic Press, 1999. 96-101

22 Yoshio S, Susumu T. Dynamic synchrony of firing in the monkey prefrontal cortex during working-memory tasks. J Neurosci, 2006, 26: 10141-10153

23 In K H, Ki Y Y, Hua L, et al. Indole-3-propionic acid attenuates neuronal damage and oxidative stress in the ischemic hippocampus. $\mathrm{J}$ Neurosci Res, 2009, 87: 2126-2137

24 Chapin J K, Moxon K A, Markowitz R S, et al. Real-time control of a robot arm using simultaneously recorded neurons in the motor cortex. Nat Neurosci, 1999, 2: 664-670 
25 Teuber H L. Unity and diversity of frontal lobe functions. Acta Neurobiol Exp(Wars), 1972, 32: 615-656

26 Fuster J M. Unit activity in prefrontal cortex during delayed-response performance: neuronal correlates of transient memory. J Neurophysiol, 1973, 36: 61-78

27 Levine S P, Huggins J E, BeMent S L, et al. A direct brain interface based on event-related potentials. IEEE Trans Rehabil Eng, 2000, 8: 180-185

28 Fuster J M, Bauer R H, Jervey J P. Cellular discharge in the dorsolateral prefrontal cortex of the monkey in cognitive tasks. Exp Neurol, 1982, 77: 679-694

29 Fuster J M, Jervey J P. Neuronal firing in the inferotemporal cortex of the monkey in a visual memory task. J Neurosci, 1982, 2: 361-375

30 Kubota K, Iwamoto T, Suzuki H. Visuokinetic activities of primate prefrontal neurons during delayed-response performance. J Neurophysiol, 1974, 37: 1197-1212

31 Sakai M. Prefrontal unit activity during visually guided lever pressing reaction in the monkey. Brain Res, 1974, 81: 297-309

32 Niki H, Watanabe M. Prefrontal unit activity and delayed response: relation to cue location versus direction of response. Brain Res, 1976, 105: 79-88

33 Boch R A, Goldberg M E. Participation of prefrontal neurons in the preparation of visually guided eye movements in the rhesus monkey. J Neurophysiol, 1989, 61: 1064-1084

Open Access This article is distributed under the terms of the Creative Commons Attribution License which permits any use, distribution, and reproduction in any medium, provided the original author(s) and source are credited. 